\title{
O Problema da Pirâmide de Base Quadrada
}

\author{
Jaime E. A. Rodriguez $^{1}, \quad$ Felipe D. C. Fidalgo ${ }^{2}$ \\ ${ }^{1}$ Depto de Matemática, FEIS, UNESP, 15385-000, Ilha Solteira, SP, E-mail: jaime@mat.feis.unesp.br \\ 2 IMECC, UNICAMP, 13083-000, Campinas, SP, E-mail: felipefidalgo@ime.unicamp.br \\ ${ }^{2}$ Universidade Estadual de Ponta Grossa, Ponta Grossa, PR
}

\begin{abstract}
Resumo: A arte de resolver problemas envolvendo equações diofantinas é muito antiga e até hoje existem problemas sem solução, ou com soluções parciais e, em outros casos, com soluções diversas. Em 1875, Edouard Lucas apresentou o seguinte problema desafiador: "Considere uma coleção de balas de canhão empilhadas em forma de pirâmide de base quadrada com uma bala na camada superior, quatro balas na segunda camada, nove balas na terceira camada e assim por diante. Se o empilhamento colapsar, é possivel reorganizar as balas em uma base quadrada?". Por longas décadas diversos matemáticos têm tentado fornecer uma solução completa (acessivel) a esse problema, e em 1985, De Gang Ma, usando idéias de Kanagasabapathy e Ponnudurai, foi o primeiro a fornecer uma solução simples e completa do problema. Neste trabalho apresentamos uma outra solução do problema de Lucas, usando as idéias de De Gang Ma.
\end{abstract}

\section{Palavras-Chave: Equação Diofantina, Reciprocidade Quadrática, Congruência.}

\section{Introdução}

Imaginemos uma coleção de balas de canhão empilhadas em forma de pirâmide de base quadrada com uma bala na camada superior, quatro balas na segunda camada, nove balas na terceira camada e assim por diante. Se o empilhamento colapsar, é possível reorganizar as balas em uma base quadrada?

Vamos analisar primeiro os casos mais simples. Se não existir nenhuma bala, se tem uma pirâmide de altura zero e um quadrado zero por zero. Se existir uma única bala, esta forma uma pirâmide de altura um e é um quadrado um por um. Além destes casos triviais, existirão outros? Se a pirâmide tiver três camadas, então o rearranjo não é possível já que existirão $1+4+9=14$ balas e 14 não é um número quadrado perfeito. O objetivo deste trabalho é apresentar uma solução geral accessível a este problema.

Em 1875, E. Lucas [7], desafiou aos leitores da revista Nouvelles Annales de Mathématique a provar o seguinte problema:

"Uma pirâmide quadrada de balas de canhão contém um número quadrado perfeito de balas de canhão unicamente se tem 24 balas de canhão ao longo de cada fileira em sua base".

Em outras palavras, Lucas afirmou que a única solução (em números naturais) da equação

$$
1^{2}+2^{2}+3^{2}+\cdots+x^{2}=y^{2}
$$

é $x=24$ e $y=70$. Aqui $x$ representa a número de balas de canhão por camada e $y$ o número total de balas, o qual deve ser um número quadrado perfeito.

Observe que a equação acima é equivalente à cúbica

$$
x(x+1)(2 x+1)=2 x^{3}+3 x^{2}+x=6 y^{2},
$$

a qual representa uma Curva Elíptica [11].

Para introduzir o problema e discutir sua solução, apresentamos a seguir uma breve história dessa equação Diofantina e na próxima seção apresentaremos a solução mencionada linhas acima. 
Em 1876, M. Moret-Blanc [9], apresentou uma "prova" da afirmação de Lucas e, embora incompleta, forneceu uma certa base para resolvê-la. M. Moret-Blanc dividiu o problema em dois casos: $x$ par e $x$ ímpar (que é o que faremos neste trabalho). Em 1877, tendo observado que existia uma "lacuna" na prova de Moret-Blanc, Lucas [8], publicou uma outra prova, a qual também tinha outra "lacuna" pois ele conseguiu lidar com o caso $x$ par mas não com o caso $x$ ímpar. Em 1918, G. N. Watson [10], conseguiu prencher a lacuna de Lucas com cerca de 14 páginas de uma Teoria Estendida de Funções Elípticas Jacobianas. Esta foi a primeira prova completa da afirmação de Lucas, mas resultou sendo muito complicada e difícil de entender na época. Em 1952, W. Ljunggren [6], usou o Método de Skolem para fornecer uma simples solução aritmética para o problema da pirâmide de base quadrada. Em 1966 [2], Baker e Davenport, usando um teorema transcendental da Teoria dos Números, conseguiram resolver as diofantinas simultâneas $3 x^{2}-2=y^{2}$ e $8 x-7=z^{2}$. Seu método, recentemente melhorado por M. Waldschmidt, pode ser adaptado para demonstrar a proposição de Lucas. Em 1975 [5], Kanagasabapathy e Ponnudurai forneceram um solução elementar para as equações diofantinas simultâneas $3 x^{2}-2=y^{2}$ e $8 x-7=z^{2}$ e, em 1985, De Gang Ma [4], obteve uma solução elementar do problema de Lucas, a primeira prova completa acessível a nível de graduação.

Neste trabalho usamos as idéias de Ma para fornecer uma outra prova da afirmação de Lucas. Na seção 2, dividida em duas partes, apresentamos a solução ao problema de Lucas.

\section{O Problema: Teoria e Resultados}

Nesta seção apresentamos uma outra solução do Problema da pirâmide de base Quadrada, dado pela equação

$$
1^{2}+2^{2}+3^{2}+\cdots+x^{2}=y^{2}
$$

com $x, y \in \mathbb{N}$. Para isso dividimos o problema em dois casos bem definidos.

\subsection{Caso I: Quando $x$ é par}

A equação original

$$
1^{2}+2^{2}+3^{2}+\cdots+x^{2}=y^{2}
$$

é equivalente a

$$
x(x+1)(2 x+1)=6 y^{2} .
$$

Nesta subseção vamos considerar o caso $x$ par. Analisaremos e resolveremos o problema com o auxílio dos seguintes lemas.

Lema 2.1. A área de um triàngulo pitagórico (retângulo) nunca é um quadrado perfeito.

Demonstração: Suponha, por absurdo, que existe um triângulo pitagórico cuja área é um quadrado. Seja $w^{2}$ a menor área para a qual tal triângulo existe. Sejam $x$ e $y$ os catetos do triângulo pitagórico com área $w^{2}$. Então $x^{2}+y^{2}=z^{2}$, para algum inteiro $z$ e $\frac{x y}{2}=w^{2}$.

Considere a terna pitagórica $(x, y, z)$. Como $w$ é minimal, então $x$ e $y$ são primos relativos e, sem perda de generalidade, podemos assumir $x$ ímpar e $y$ par (e portanto, $z$ ímpar). De fato, se ambos, $x$ e $y$, fossem ímpares, então $z$ seria par e, portanto,

$$
z^{2} \equiv 0(\bmod 4) \quad \text { e } \quad x^{2} \equiv y^{2} \equiv 1(\bmod 4)
$$

o que contraria a igualdade $x^{2}+y^{2}=z^{2}$.

Agora afirmamos que existem inteiros primos relativos $r$ e $s$, de paridade diferente, tais que

$$
x=r^{2}-s^{2} \quad \text { e } \quad y=2 r s .
$$

De fato, dado que temos um triângulo pitagórico primitivo (pois $m d c(x, y)=1$ ), então da igualdade $x^{2}+y^{2}=z^{2}$ obtemos $y^{2}=(z+x)(z-x)$, ou seja, $\left(\frac{y}{2}\right)^{2}=\left(\frac{z+x}{2}\right)\left(\frac{z-x}{2}\right)$. Observe 
que $z+x$ e $z-x$ são números pares. Agora, vamos mostrar que $\operatorname{mdc}\left(\frac{z+x}{2}, \frac{z-x}{2}\right)=1$. Seja $d=m d c\left(\frac{z+x}{2}, \frac{z-x}{2}\right)$. Então

$$
\left\{\begin{array}{c}
\mathrm{d} \text { divide } \frac{z+x}{2} \\
\mathrm{~d} \text { divide } \frac{z-x}{2}
\end{array}\right.
$$

e portanto

$$
\left\{\begin{array}{c}
\mathrm{d} \text { divide } \frac{z+x}{2}+\frac{z-x}{2}=z \\
\mathrm{~d} \text { divide } \frac{z+x}{2}-\frac{z-x}{2}=x .
\end{array}\right.
$$

Assim, dado que $m d c(z, x)=1$, então $d=1$.

Segue então que existem inteiros positivos $r$ e $s$ tais que $\frac{z+x}{2}=r^{2} e \frac{z-x}{2}=s^{2}$, de onde obtemos que $y=2 r s$ e $x=r^{2}-s^{2}$ (subtraindo as duas igualdades acima). Assim mostramos a afirmação mencionada linhas acima.

Segue desta afirmação que $\left(r^{2}-s^{2}\right) r s=w^{2}$ e que $s / 4<s \leq w^{2}$. Já que $r, s, r-s$ e $r+s$ são primos relativos dois a dois e como $(r-s)(r+s) r s=w^{2}$, segue que existem inteiros positivos $a, b, c$ e $d$ tais que $r=a^{2}, s=b^{2}, a^{2}-b^{2}=r-a=c^{2}$ e $a^{2}+b^{2}=r+s=d^{2}$. Observar que $m d c(c, d)=1$, pois $m d c(r-s, r+s)=1$. Observe também que $c$ e $d$ são ímpares pois $r$ e $s$ tem paridade diferente.

Agora, consideremos $X=\frac{d+c}{2}$ e $Y=\frac{c-d}{2}$. Então $X$ e $Y$ são primos relativos e $X^{2}+Y^{2}=$ $a^{2}$. Portanto ou $X$ ou $Y$ é par e $\frac{X Y}{2}=\frac{d^{2}-c^{2}}{8}=\frac{b^{2}}{4}=\frac{s}{4}$ é um quadrado. Dado que o triângulo de lados $X, Y$ e a é um triângulo pitagórico com área $s / 4$, segue da minimalidade de $w^{2}$ que $w^{2} \leq s / 4$, o qual é uma contradição. Assim fica demonstrado o lema.

Lema 2.2. Não existe nenhum inteiro positivo $x$ tal que $2 x^{4}+1$ seja um quadrado.

Demonstraçâo: Por absurdo, suponha que $(x, y)$ seja a menor solução inteira da equação $2 x^{4}+1=y^{2}$. Então para algum inteiro positivo $s$ temos que $y=2 s+1$, de onde obtemos que $x^{4}=2 s(s+1)$. Se $s$ for ímpar então $m d c(s, 2(s+1))=1$ e assim, para alguns inteiros $u$ e $v$ temos que $s=u^{4}$ e $2(s+1)=v^{4}$. Segue então que $2\left(u^{4}+1\right)=v^{4}$, com $u$ ímpar e $v$ par. Portanto, temos $2(1+1) \equiv 0(\bmod 8)$, o que é impossível. Sendo assim $s$ não pode ser ímpar. Logo $s$ é par. Segue então que $m d c(2 s, s+1)=1$ e portanto existem inteiros $u$ e $v$, ambos maiores do que 1 , tais que $2 s=u^{4}$ e $s+1=v^{4}$.

Seja $w$ o inteiro positivo tal que $u=2 w$. Seja $a$ o inteiro positivo tal que $v^{2}=2 a+1$. Então $\frac{u^{4}}{2}+1=s+1=v^{4}$, de modo que $2 w^{4}=\frac{v^{4}-1}{4}=a(a+1)$. Como $v^{2}=2 a+1$, segue que $a$ é par. Agora, como $2 w^{4}=a(a+1)$, segue-se que existem inteiros positivos $b$ e $c$ tais que $a=2 b^{4}$ e $a+1=c^{4}$. Porém isto implica que $2 b^{4}+1=\left(c^{2}\right)^{2}$ (ou seja, $c^{2}$ é uma solução da equação $\left.2 x^{4}+1=y^{2}\right)$ e portanto $y \leq c^{2}$, pela minimalidade de $(x, y)$. Por outro lado temos que $c^{2} \leq a+1<v^{2} \leq s+1<y$, o que é uma contradição. Assim fica mostrado o lema.

Lema 2.3. Existe exatamente um único inteiro positivo $x=1$, tal que $8 x^{4}+1$ é um quadrado.

Demonstração: Suponha que $8 x^{4}+1=(2 s+1)^{2}$, para algum $s$ inteiro positivo. Então obtemos que $2 x^{4}=s(s+1)$.

Se $s$ for par então existem inteiros $u$ e $v$ tais que $s=2 u^{4}$ e $s+1=v^{4}$. Neste caso $2 u^{4}+1=v^{4}$ e pelo Lema 2.2 , se tem $u=0$ e portanto, $x=0$. 
Se $s$ for ímpar então existem inteiros positivos $u$ e $v$ tais que $s=u^{4}$ e $s+1=2 v^{4}$. Neste caso $u^{4}+1=v^{4}$. Como $u$ é ímpar, resulta $v$ ímpar. Elevando ao quadrado ambos os lados da expressão $u^{4}+1=2 v^{4}$ obtemos $4 v^{8}-4 u^{4}=u^{8}-2 u^{4}+1$ e portanto

$$
\left(v^{4}-u^{2}\right)\left(v^{4}+u^{2}\right)=\left(\frac{u^{4}-1}{2}\right)^{2}
$$

que é um quadrado perfeito.

Como $m d c\left(u^{4}, v^{4}\right)=1$, segue que $\frac{v^{4}-u^{2}}{2}$ e $\frac{v^{4}+u^{2}}{2}$ são quadrados perfeitos. Agora sejam

$$
\left(v^{2}-u\right)^{2}+\left(v^{2}+u\right)^{2}=4 \frac{v^{4}+u^{2}}{2}=A^{2} \quad e \quad \frac{\left(v^{2}-u\right)\left(v^{2}+u\right)}{2}=\frac{v^{4}-u^{2}}{2}=B^{2} .
$$

Mas pelo lema 2.1 isto é impossível, a menos que $v^{2}= \pm u$. E como $u^{4}+1=2 v^{4}$, obtemos $u^{4}-2 u^{2}+1=\left(u^{2}-1\right)^{2}=0$, de onde $u^{2}=1$. Daqui segue que $s=1$ e $x= \pm 1$.

Agora, com o auxílio dos três lemas acima, estamos em condições de resolver a equação

$$
x(x+1)(2 x+1)=6 y^{2},
$$

sob a hipótese de $x$ ser par.

Se $x$ é par então $x+1$ é ímpar. Como $x, x+1$ e $2 x+1$ são primos relativos dois a dois, segue que $x+1$ e $2 x+1$ (sendo ambos ímpares) são ou quadrados perfeitos ou três vezes um quadrado perfeito. Assim

$$
x+1 \nsucceq 2(\bmod 3) \quad e \quad 2 x+1 \nsucceq 2(\bmod 3) .
$$

Portanto $x \equiv 0(\bmod 3)$, e existem inteiros não negativos $p, q$ e $r$ tais que

$$
x=6 q^{2}, x+1=p^{2} \text { e } 2 x+1=r^{2} .
$$

Assim $6 q^{2}=(r-p)(r+p)$. Como $p$ e $q$ são ambos ímpares, então o 4 é um fator de $(r-p)(r+p)=6 q^{2}$ e assim $q$ é par. Seja $q_{0}$ um inteiro tal que $q=2 q_{0}$. Agora se tem

$$
6 q_{0}^{2}=\left(\frac{r-p}{2}\right)\left(\frac{r+p}{2}\right),
$$

e como $m d c\left(\frac{r-p}{2}, \frac{r+p}{2}\right)=1$ (pois $m d c\left(r^{2}, p^{2}\right)=1$ ), obtemos um dos seguintes casos:

Caso 1: Das expressões $\frac{r-p}{2}$ e $\frac{r+p}{2}$, uma é da forma $6 A^{2}$ e a outra da forma $B^{2}$, onde $A$ e $B$ são inteiros não negativos. Então $p= \pm\left(6 A^{2}-B^{2}\right)$ e $q=2 A B$. Como $6 q^{2}+1=x+1=p^{2}$, temos $24 A^{2} B^{2}+1=\left(6 A^{2}-B^{2}\right)^{2}$ ou $\left(6 A^{2}-3 B^{2}\right)^{2}-8 B^{4}=1$. Logo, pelo Lema $2.3, B=0$ ou $B=1 \mathrm{e}$, portanto, $x=6 q^{2}=0$ ou $x=24$. Assim a única solução não trivial é $x=24$.

Caso 2: Das expressões $\frac{r-p}{2}$ e $\frac{r+p}{2}$, uma é da forma $3 A^{2}$ e a outra da forma $2 B^{2}$, onde $A$ e $B$ são inteiros não negativos. Então $p= \pm\left(3 A^{2}-2 B^{2}\right)$ e $q=2 A B$. Isto dá $24 A^{2} B^{2}+1=\left(3 A^{2}-2 B^{2}\right)^{2}$ e, portanto, $\left(3 A^{2}-6 B^{2}\right)^{2}-2(2 B)^{4}=1$. Logo, pelo Lema 2.2 se tem $B=0$ e assim $x=6 q^{2}=0$.

Desta forma, quando $x$ é par, a única solução do enigma de Lucas é $x=24$ balas de canhão ao longo da base da pirâmide quadrada e, portanto, $y=70$. 


\subsection{Caso II: Quando $x$ é ímpar}

Para resolver o problema nesta segunda parte, primeiro investigaremos as soluções da equação Diofantina $X^{2}-3 Y^{2}=1$. Para isso sejam $a=2+\sqrt{3}$ e $b=2-\sqrt{3}$. Observar que $a b=1$. Para qualquer $n$ inteiro não-negativo, sejam

$$
u_{n}=\frac{a^{n}+b^{n}}{2} \quad e \quad v_{n}=\frac{a^{n}-b^{n}}{2} .
$$

Então $u_{n}$ e $v_{n}$ são inteiros e, quando $n \geq 1,\left(u_{n}, v_{n}\right)$ é a $n$-ésima solução inteira da equação $X^{2}-3 Y^{2}=1$. De fato, quando $n=0$, temos a solução $X=1$ e $Y=0$.

Agora, a fim de mostrar que $x=1$ é o único inteiro positivo ímpar tal que $x(x+1)(2 x+1)$ é da forma $6 y^{2}$, faremos uso dos lemas seguintes.

Lema 2.4. Se $m$ e $n$ são inteiros não-negativos, então:

(a) $u_{m+n}=u_{m} u_{n}+3 v_{m} v_{n}$ e $v_{m+n}=u_{m} v_{n}+u_{n} v_{m}$.

(b) Se $m-n \geq 0$, então $u_{m-n}=u_{m} u_{n}-3 v_{m} v_{n}$ e $v_{m-n}=-u_{m} v_{n}+u_{n} v_{m}$.

Demonstração: Ambas as partes seguem de cálculos diretos das definições de $u_{n}$ e e $v_{n}$.

Usando o lema acima, não é difícil obter o seguinte resultado.

Lema 2.5. Se $m$ é um inteiro não-negativo, então $u_{m+2}=2 u_{1} u_{m+1} \quad e \quad v_{m+1}=2 u_{1} v_{m+1}-v_{m}$.

Agora, usando o fato de que $\left(u_{m}, v_{m}\right)$ é a solução da equação $X^{2}-3 Y^{2}=1$, temos também o seguinte resultado.

Lema 2.6. Se $m$ é um inteiro não-negativo, então $u_{2 m}=2 u_{m}^{2}-1=6 v_{m}^{2}+1$ e $v_{2 m}=2 u_{m} v_{m}$.

Lema 2.7. Sejam $m, n$ e $r$ inteiros não-negativos tais que $2 r m-n$ seja também inteiro nãonegativo. Então $u_{2 r \pm n} \equiv(-1)^{r} u_{n}\left(\bmod u_{m}\right)$.

Demonstração: Usamos indução sobre $r$ e o Lema 2.4. Verifica-se que

$$
u_{(2 r+1) m} \equiv 0\left(\bmod u_{m}\right) \quad \text { e } v_{2 r m}=\equiv 0\left(\bmod u_{m}\right) .
$$

Ja que o lema 2.6 implica que $u_{2 r m}=2 u_{r m}^{2}-1=6 v_{r m}^{2}+1$, segue que $u_{2 r m} \equiv(-1)^{r}\left(\bmod u_{m}\right)$. Desta forma, pelo Lema 2.4, obtemos

$$
u_{2 r m \pm n}=u_{2 r m} u_{n} \pm 3 v_{2 r m} v_{n} \equiv(-1)^{r}\left(\bmod u_{m}\right) .
$$

Agora consideremos alguns poucos valores de $u_{n}$. Começando por $n=0$, temos 1, 2, 7, 26, 97, $362, \cdots$ e assim por diante. Se consideramos estes valores, módulo 5 , teremos $1,2,2,1,2,2, \cdots$. Pelo Lema 2.5, esta é uma sequência periódica de período 4. Observe que quando $n$ é par, $u_{n}$ é ímpar.

A Lei de Reciprocidade Quadrática é um teorema acerca da Aritmética Modular que fornece condições para a resolubilidade de equações quadráticas módulo números primos. Dado $p$ primo, essa lei fornece uma bela descrição de quais números primos são quadrados módulo $p$. Casos especiais desta lei se devem a Fermat, Euler e Legendre, mas foi Gauss quem apresentou a primeira prova completa. Explicitamente, a Lei da Reciprocidade Quadrática estabelece que se $p$ e $q$ são primos (ímpares) distintos, sendo pelo menos um deles congruente a 1 módulo 4 , então $p$ é um quadrado módulo $q$ se, e somente se, $q$ é um quadrado módulo $p$. Para estabelecer tal lei usa-se o Simbolo de Legendre. 
Definicão 2.1. (Símbolo de Legendre) Seja $p$ um número primo e a um número inteiro tal que $m d c(a, p)=1$. Então o símbolo $\left(\frac{a}{p}\right)$ é definido por:

$$
\left(\frac{a}{p}\right)=\left\{\begin{array}{l}
1, \text { se } n \text { é um resíduo quadrático } \bmod p \\
-1, \text { se n é um não-resíduo quadrático } \bmod p \\
0, \text { se } p \mid a .
\end{array}\right.
$$

Usando a Lei de Reciprocidade Quadrática, os comentários acima nos levam a obter os seguintes dois resultados.

Lema 2.8. Se n é par então $u_{n}$ é um ímpar não múltiplo de 5 e $\left(\frac{5}{u_{n}}\right)=1$ se, e somente se, $n$ é um múltiplo de 3.

Lema 2.9. Se n é par então $u_{n}$ é ímpar e $\left(\frac{-2}{u_{n}}\right)=1$ se, e somente se, $n$ é um múltiplo de 4.

O seguinte resultado foi mostrado por De G. Ma [4].

Lema 2.10. Se $n$ é um inteiro não negativo então $u_{n}$ tem a forma $4 M^{2}+3$ unicamente quando $u_{n}=7$.

Demonstração: Suponha $u_{n}=4 M^{2}+3$. Então $u_{n} \equiv 3$ ou $7(\bmod 8)$ e, da sequência de valores de $u_{n}$ módulo 8 , segue que $n$ tem a forma $8 k \pm 2$. Agora suponha que $n \neq 2$ (e, portanto, $u_{n} \neq 7$ ). Então podemos escrever $n$ na forma $2 q 2^{s} \pm 2$, onde $q$ é ímpar e $s \geq 2$. Assim, pelo Lema 2.7 , se tem $u_{n}=u_{2 q 2^{s} \pm 2} \equiv(-1)^{q} u_{2}\left(u_{2^{s}}\right)$ e portanto

$$
\left(\frac{-2}{u_{2}}\right)\left(\frac{5}{u_{2}}\right)=\left(\frac{-10}{u_{2}}\right)\left(\frac{4 M^{2}}{u_{2}}\right)=1 .
$$

Assim, do Lema 2.9 segue que o primeiro fator da esquerda vale 1 e do Lema 2.8 segue que o segundo fator da esquerda é -1 . Como isto é impossível, concluímos que $n=2 \mathrm{e}$, portanto, $u_{n}=7$.

Agora, suponha que $x$ é inteiro ímpar positivo e $x(x+1)(2 x+1)=6 y^{2}$, para algum inteiro $y$. Dado que $x, x+1$ e $2 x+1$ são primos relativos, dois a dois, segue que $x$ é um quadrado ou o triplo de um quadrado, e portanto, $x \nsubseteq 2(\bmod 3)$. Mais ainda, como $x+1$ é par, então é o dobro de um quadrado perfeito ou seis vezes um quadrado perfeito e, portanto, $x+1 \not 1(\bmod 3)$. Sendo $\operatorname{assim} x \equiv 1(\bmod 3)$ e, portanto, $x+1 \equiv 2(\bmod 3)$ e $2 x+1 \equiv 0(\bmod 3)$. Assim, para alguns inteiros não negativos $u, v$ e $w$ se tem que $x=u^{2}, x+1=2 v^{2}$ e $2 x+1=3 w^{2}$. Segue disto que $6 w^{2}+1=4 x+3=4 u^{2}+3$. Também temos que

$$
\left(6 w^{2}+1\right)^{2}-3(4 v w)^{2}=12 w^{2}\left(3 w^{2}+1-4 v^{2}\right)+1=12 w^{2}(2 x+1+1-2(x+1))+1=1 .
$$

Logo, pelo Lema 2.10, se tem que $6 w^{2}+1=7$. Assim $w=1$ e $4 x=1$. Isto fornece a solução trivial (1) de balas de canhão para o problema de Lucas.

Concluimos então que se um número quadrado de balas de canhão são dispostas em uma pirâmide de base quadrada, então existem exatamente 4900 delas.

\section{Referências Bibliográficas}

[1] W. S. Anglin, The Square Pyramid Puzzle, The American Mathematical Monthly, Vol. 97, No. 2, 1990, pp. 120-124. 
[2] A. Baker and H. Davenport, The equations $3 x^{2}-2=y^{2}$ and $8 x^{2}-7=z^{2}$, Quaterly Journal of Mathematics, ser. 2, 20 (1969), 129-37.

[3] I, Cucurezeanu, An Elementary Solution of Luca's Problem, Journal of Number Theory, 44, 9-12 (1993).

[4] De Gang Ma, An Elementary proof of the solution to the Diophantine Equation $6 y^{2}=$ $x(x+1)(2 x+1)$, Sichuan Daxue Xuebao, 4 (1985), 107-16.

[5] P. kanagasabapathy and T. Ponnudurai, The simultaneous Diophantine equations $y^{2}-$ $3 x^{2}=-2$ and $z^{2}-8 x^{2}=-7$, Quarterly Journal of Mathematics, ser. 2, 26 (1975), 275-78.

[6] W. Ljunggren, New Solution of a Problem proposed by E. Lucas, Norsk Mat. Tidsskrift, 34 (1952), 65-72.

[7] E. Lucas, Question 1180, Nouvelles Annales de Mathématiques, ser. 2 , 14 (1875), 336.

[8] E. Lucas, Question 1180, Nouvelles Annales de Mathématiques, ser. 2, 16 (1877), 429-32.

[9] M. Moret-Blanc, Question 1180, Nouvelles Annales de Mathématiques, ser. 2, 15 (1876) $46-48$

[10] G. N. Watson, The problem of the square pyramid, Messenger of Mathematics, 48 (1918$19), 1-22$.

[11] L. C. Washington, Elliptic Curves, Number Theory and Cryptography, CRC Press, Taylor and Francis Group, Second Edition, 2008. 